\title{
Lineamientos para una propuesta de política pública de recuperación productiva: Manabí 2016
}

\author{
Elisa Lanas Medina* \\ Genoveva Espinoza Santeli**
}

\section{RESUMEN}

El terremoto de 2016, con epicentro en Manabí generó la oportunidad para analizar si el Ecuador cuenta con políticas públicas para la gestión del riesgo, analizar la institucionalidad de los mecanismos para responder durante y después de un desastre, y si la gestión posdesastre incluye políticas para reactivar las actividades productivas. Es una investigación mixta, con alcance exploratorio y descriptivo, con el objetivo de proponer lineamientos para la política pública en gestión de posdesastre con procesos alternativos de producción, basados en la economía solidaria, como mecanismos de recuperación.

El hallazgo más significativo es que el país no cuenta con políticas públicas adecuadas, las instituciones encargadas de la gestión del riesgo son débiles, y la sociedad no es resiliente; además, se identificó que es preciso realizar un diagnóstico de la realidad del territorio para esbozar los lineamientos para proponer la política pública de recuperación productiva mediante procesos asociativos y solidarios.

PALABRAS CLAVE: desastres naturales, política pública, economía solidaria, organizaciones sin fines de lucro.

\section{ABSTRACT}

The 2016 earthquake, with an epicenter in Manabí, created the opportunity to analyze if Ecuador has risk management public policies, analyze the institutional framework of the mechanisms to respond during and after a disaster and, if the post-disaster management includes policies to reactivate productive activities. It is a mixed research, with exploratory and descriptive scope and the aim of proposing guidelines for public policy in post-disaster management with alternative production processes, based on solidarity economy, as a recovery mechanisms.

\footnotetext{
* Docente investigadora del Área de Derecho de la Universidad Andina Simón Bolívar, Sede Ecuador.
}

** Docente investigadora del Área de Gestión de la Universidad Andina Simón Bolívar, Sede Ecuador. 
The most significant finding is that Ecuador does not have adequate public policies for risk management, the institutions are weak and the community is not resilient; also, it was identified that it is necessary to make a diagnosis of the reality of the territory to outline the guidelines to propose the public policy of productive recovery through associative and solidarity processes.

KEYWORDs: natural disasters, public policy, solidarity economy, non-profit organizations.

\section{INTRODUCCIÓN}

$\mathrm{E}$ 116 de abril de 2016 a las 18:58 el Ecuador sufrió un terremoto con una magnitud de 7,8 grados en la escala de Richter seguido de 2.709 réplicas hasta el 27 de octubre del mismo año. El epicentro del evento estuvo situado entre las parroquias de Pedernales y Cojimíes, provincia de Manabí, y el movimiento telúrico también se sintió y afectó a otras zonas y provincias del país, como Guayas, Santa Elena, Los Ríos, Santo Domingo y El Oro en la Costa, y en provincias de la Sierra como Carchi, Imbabura y Pichincha. Este suceso está considerado como el más destructivo desde el terremoto registrado en 1987 que tuvo una magnitud de 6,9 grados en la escala de Richter, con epicentro en el noroeste de Ecuador, en las provincias de Sucumbíos y Napo.

El terremoto de 2016, según cifras oficiales de la Secretaría de Gestión de Riesgos del Ecuador, dejó 663 personas fallecidas, 9 personas desaparecidas, 6.274 personas heridas y con otras afectaciones directas, 113 personas rescatadas con vida, y 28.775 personas distribuidas en 37 albergues y 214 refugios.

En términos económicos, "Ecuador necesitaría más de 3.300 millones de dólares para obras de reconstrucción, lo que implica un decrecimiento de $0,7 \%$ del PIB del año anterior". ${ }^{1}$ Para la Federación Ecuatoriana de Exportadores, un total de $150 \mathrm{mi}-$ llones de dólares se dejaron de exportar considerando solo la producción de empresas exportadoras localizadas en la provincia de Manabí y Esmeraldas, y en términos macroeconómicos representó el 8\% de las exportaciones totales a nivel nacional.

1. “Dos fuertes sismos sacuden Ecuador con epicentro en zona terremoto de abril”. El Nuevo Herald (Miami). 11 de julio de 2016. Consulta: febrero de 2017. Disponible en «http://www.elnuevoherald.com/noticias/mundo/americalatina/article88838672.html\#storylink=cpy». 
El Banco Interamericano de Desarrollo, con un modelo de regresión realizó un análisis de dos mil (2000) desastres naturales ocurridos entre 1970 y 2008, utilizando variables como la población del país, el PIB per cápita, el área del país, el PIB real y el número de fallecidos para determinar el costo del desastre, ${ }^{2}$ obteniendo que para Ecuador el costo del terremoto estaría entre 500 y 600 millones de dólares. La agencia United States Geological Survey también realizó algunas estimaciones, indicando que las pérdidas económicas ascenderían al menos a mil millones. Por su parte, las fuentes oficiales del gobierno, después de realizar una valoración del costo mediante una metodología internacional de las Naciones Unidas, denominada "Evaluación Posdesastres de Necesidades", estima que el costo de la recuperación estaría en el rango de dos mil y tres mil millones de dólares, el equivalente al 2, 3\% del PIB de 2016.

Concretamente en el sector productivo, según estimaciones de la SENPLADES, ${ }^{3}$ los costos de reconstrucción de este sector ascienden a los mil treinta y dos millones de dólares, siendo los subsectores afectados: el comercio en un 44\%; agricultura, ganadería, acuacultura y pesca en un $31 \%$; manufactura en un $16 \%$; y servicios turísticos en un $9 \%$. De esas afectaciones, el $22 \%$ corresponde al sector público y el $78 \%$ al sector privado, lo que indica que en el sector productivo son los propios afectados quienes deben encargarse de la recuperación.

Frente a este escenario de grandes pérdidas económicas, humanas, sociales y ambientales, y a más de un año del terremoto, se analizó la respuesta del gobierno ecuatoriano ante los desastres, y si es preciso plantear una política pública de largo plazo, para que, en la medida de lo posible, las personas recuperen su modo de vida en todos los aspectos, siendo la actividad productiva una de las más importantes, ya que "para las personas que perdieron tanto, no solo el trabajo, también a sus seres queridos, el trabajo les ayuda no solo a recuperar un sustento, también a superar el dolor, la desesperación y aporta esperanza". ${ }^{4}$

El objetivo de este artículo es plantear los lineamientos de una política pública para la recuperación productiva de un territorio, en este caso Manabí, mediante procesos asociativos y solidarios, después de un desastre.

2. Eduardo Cavallo, Andrew Powell y Oscar Becerra, "Estimating the Direct Economic Damages of the Earthquake in Haiti", The Economic Journal, No. 546 (2010): 298-312.

3. SENPLADES, Evaluación de los costos de reconstrucción sismo en Ecuador. Abril 2016. Consulta: febrero de 2017. Disponible en 〈http:/www.planificacion.gob.ec/wp-content/uploads/downloads/2017/04/ Evaluacion-de-los-Costos-de-Reconstruccion-Libro-Completo.pdf $\rangle$.

4. "Volver a la normalidad después del gran terremoto que azotó el este de Japón". Organización Internacional del Trabajo, 2013. Consulta: mayo de 2017. Disponible en «http://www.ilo.org/global/about-the-ilo/ newsroom/features/WCMS_208802/lang--es/index.htm〉. 
Con ese fin, se presenta un marco teórico y legal de respuesta al desastre, que servirá como sustento para elaborar un diagnóstico con miras a proponer políticas públicas adecuadas en materia de reactivación económica, que se afiance principalmente en alianzas estratégicas público-privadas para promover las iniciativas de la economía solidaria y del cooperativismo en la ardua tarea de recuperación de las zonas afectadas, concretamente de la provincia de Manabí.

\section{MARCO TEÓRICO}

Para poder cumplir con el objetivo de la investigación, que es aportar con lineamientos para una política pública de recuperación productiva en la provincia de Manabí que fomente la economía solidaria, es necesario rescatar los conceptos nucleares del problema de investigación. En un primer momento, se explican los procesos y procedimientos necesarios para lograr una recuperación integral en el corto, mediano y largo plazo, y posteriormente se desarrolla y explica cómo la economía solidaria puede ser una respuesta eficaz para la reactivación económica de la zona.

\section{Respuesta Al Desastre}

Un desastre es una seria interrupción en el funcionamiento de una comunidad que ocasiona muertes, pérdidas e impactos materiales, económicos y ambientales, que exceden la capacidad de respuesta de una comunidad. ${ }^{5}$

La gestión del riesgo de desastres es "el proceso sistemático de utilizar directrices administrativas, organizaciones, destrezas y capacidades operativas para ejecutar políticas y fortalecer las capacidades de afrontamiento con el fin de reducir el impacto adverso de las amenazas naturales y la posibilidad de que ocurra un desastre".${ }^{6}$ Busca la recuperación integral de la zona a través de los procesos de rehabilitación y de reconstrucción.

La recuperación integral debe estar enmarcada en un modelo de planificación en el que el ordenamiento territorial y la construcción desde las comunidades locales sea

5. Allan Lavell, "Sobre la gestión del riesgo: Apuntes hacia una definición", Scripta Nova-Revista (2001). Consulta: marzo de 2017. Disponible en 〈http://microseguros.net/seminario/biblioteca/vulnerabilidad/ doc15036-lavell-gestion-riesgo.pdf>.

6. “Annual Report 2015 2014-15 Biennium Work Programme Final Report”. Oficina de las Naciones Unidas para la Reducción del Riesgo de Desastres (Ginebra). Consulta: junio de 2017. Disponible en 〈http:// www.unisdr.org/files/48588_unisdrannualreport2015evs.pdf〉. 
una estrategia ${ }^{7}$ que se conjuga con la economía solidaria para construir territorios resilientes. La rehabilitación es una estrategia a corto plazo, que implica volver a poner en funcionamiento instalaciones vitales, estructuras físicas y estructuras de servicios esenciales, ${ }^{8}$ y la reconstrucción consiste en un proceso a mediano y largo plazo, dependiendo de la infraestructura previa y el nivel de daños ocasionados por el desastre, así como de las estrategias y políticas preexistentes. El nivel de impacto se determinará en varios momentos - después de la revisión inicial y en el momento del diseño de programas nacionales, privados y mixtos; actividades que entran en la reconstrucción pueden ser la dotación o rehabilitación de alcantarillados- dependiendo del nivel de daño, infraestructura de utilidad pública, carreteras, legalización de tierras, recuperación de espacios públicos, planificación territorial, planificación de contingencias, elaboración de un plan para la reducción del riesgo de desastres, que incluya estrategias de mitigación, preparación y prevención, desarrollo de capacidades de resiliencia entre la comunidad, desarrollo de un sistema de alerta temprana, entre otras. ${ }^{9}$

La recuperación integral, después de un desastre, es vista por muchos como una lucha en contra de la madre naturaleza para restablecer el orden en la comunidad. Sin embargo, el proceso de recuperación no es una serie de pasos que debe darse automáticamente después de ocurrido el desastre; debe ser entendido como un proceso, una serie de actividades sin un orden estricto que debe darse antes, durante y después del desastre. ${ }^{10}$ Es un momento de oportunidad para investigar el modelo de desarrollo económico empleado en la región, y en el momento del diseño de los programas y proyectos de reconstrucción y recuperación se deben discutir elementos del desarrollo sustentable en aras de mejorar la calidad de vida de los damnificados, replantear las actividades y la productividad para que sean sostenibles y sustentables, protegiendo y conservando el medioambiente.

7. Juan Márquez Domínguez y Adriano Rovira Pinto, "El desarrollo local: una propuesta social y económica con fuerte base territorial", Espacio y Desarrollo, No. 14 (2002): 47-57.

8. Tales como las redes eléctricas, provisión de agua y telecomunicaciones, puertos, transporte, hospitales, bomberos, policía y administración pública. Luis Carrio Carro, "El concepto de resiliencia y su aplicación en Avilés" (tesis de maestría, Universidad de Oviedo, Máster Universitario en Análisis y Gestión de Emergencia y Desastre, 2015). Disponible en 〈http://digibuo.uniovi.es/dspace/handle/10651/32011〉.

9. Jorge Enrique Vargas, Políticas públicas para la reducción de la vulnerabilidad frente a los desastres naturales y socio-naturales, Serie medio ambiente y desarrollo, No. 50 (Santiago de Chile: CEPAL, 2002).

10. Ruth Jackson, Daniel Fitzpatrick y Prabin Man Singh, Building Back Right: Ensuring Equality in Land Rights and Reconstruction in Nepal (Oxford: Oxfam, 2016). Disponible en «http://policy-practice.oxfam. org.uk/publications/building-back-right-ensuring-equality-in-land-rights-and-reconstruction-in-nepal-606028>. 
A través de una breve sistematización de los procesos y actividades que los países deberían tener institucionalizados en caso de atravesar por un desastre, ${ }^{11}$ se elaboró una lista, con su consecuente explicación por proceso, de las actividades que un país debe poner en funcionamiento para lograr una recuperación integral, rehabilitación o reconstrucción de la zona. Estos procesos deben estar contemplados en una política pública o en un plan de riesgos y desastres, y es alrededor de estos procesos que se realizó el barrido de información en el ordenamiento jurídico ecuatoriano.

1. Ayuda humana inmediata y emergente.

2. Evacuación en las zonas de riesgo.

3. Provisión de refugios temporales.

4. Búsqueda y rescate.

5. Evaluación de daños.

6. Limpieza, remoción y disposición de escombros.

7. Restauración de servicios básicos y de comunicación.

8. Restablecimiento de las principales redes de transporte.

9. Habilitación de albergues.

10. Provisión de recursos financieros.

11. Análisis del impacto económico.

12. Inspección detallada de las edificaciones e instalaciones de propiedad privada y pública.

13. Planificación de desarrollo.

14. Evaluación ambiental.

15. Demolición de infraestructura insegura e inestable.

16. Reconstrucción.

17. Mitigación de riesgos.

18. Implementación de un sistema de alerta temprana para zonas propensas a los desastres.

19. Desarrollo de legislación pertinente.

20. Preparación para el siguiente desastre.

Estas actividades, sin llegar a ser una lista exhaustiva, deben ir atravesadas por varios procesos como: 1) desarrollo de capacidades, 2) coordinación de actividades, 3)

11. Se revisaron las sugerencias de varios autores (Bagai 2005; Adie 2001; Villanueva Haro 2007; Natt 2011; Vargas 2002; Jackson 2016 y Carter 2016). 
planificación urbana, 4) involucramiento de la comunidad, 5) información, 6) gobernanza, 7) evaluación del daño, y 8) reactivación económica, los cuales son explicados a continuación.

1. El desarrollo de capacidades es el proceso mediante el cual la población combina todas sus fortalezas, atributos y recursos para lograr los objetivos formulados, debe ir acompañada de una capacidad de afrontamiento ante condiciones adversas o situaciones de emergencia; las capacidades pueden incluir tanto medios físicos como habilidades y destrezas, y se requiere de una concientización continua. Las comunidades que están seriamente comprometidas con la disminución de riesgos antes de un desastre, por lo general, se centran en operaciones y desarrollo de capacidades de prevención, evacuación, restablecimiento de energía.

2. Uno de los retos más significativos es coordinar las actividades para que no se den situaciones como: superposición o duplicación de acciones y uso ineficiente de recursos; tomar decisiones rápidas que podrían afectar el bienestar de la comunidad a largo plazo; no tomar en cuenta la materialidad de todos los actores; perder oportunidades para mejorar la infraestructura, reactivación económica, protección del medioambiente y mejoramiento de la calidad de vida.

3. La planificación urbana es el eje fundamental para la recuperación, rehabilitación y reconstrucción de cualquier ciudad o zona después de haber sufrido un desastre natural. Para Villanueva dentro de la planificación urbana hay que considerar tres ejes fundamentales:

a) Los sistemas vitales de redes de agua, desagüe, electricidad, comunicaciones, transporte y seguridad;

b) Instalaciones de centros de salud, albergues, unidades educativas primarias y secundarias, depósitos de alimentos. Se debe analizar y considerar la seguridad de las edificaciones; $y$, en el caso de tener que reconstruir edificios y viviendas, reflexionar sobre aspectos como la compatibilidad de uso, diseño, tecnología, materiales, impacto ambiental y legislación pertinente; $y$,

c) Centros de coordinación y colaboración de actividades y proyectos públicos y privados. ${ }^{12}$

En la planificación territorial se deben considerar suficientes espacios comunales, tierra para construcción de viviendas sociales, planificar el crecimiento

12. Benito Villanueva Haro, "Terremoto en Ica-Perú: Por una reconstrucción con rostro social”, Derecho y Cambio Social, No. 11 (2007). Disponible en 〈http://www.derechoycambiosocial.com/revista011/terremoto $\% 20$ en $\% 20$ ica.htm>. 
demográfico, cuidar las fuentes de agua, redes de alcantarillado y los lugares adecuados para la gestión de residuos sólidos. La elaboración de la planificación territorial y la selección de los objetivos de recuperación y reparación deben ser tratados y discutidos previamente con la comunidad, es decir, debe haber un desarrollo participativo de todos los proyectos, considerando los factores sociales, económicos, ecológicos, culturales y jurídicos para garantizar la calidad, pertinencia viabilidad y sostenibilidad de los proyectos

4. Por lo tanto, el proceso ideal de recuperación de un desastre, según Adie, se da cuando la comunidad apoya proactivamente al proceso de identificación de objetivos comunes, ${ }^{13}$ hay un involucramiento directo en el cual se identifican los obstáculos, se toman en cuenta los principios de la sustentabilidad y sostenibilidad en el desarrollo de los proyectos, se adoptan estrategias que buscan una reparación integral de la zona, existe un consenso en los marcos regulatorios, normativos y procesales, existe coordinación, liderazgo y personas capacitadas y comprometidas para llevar a cabo el proceso de gestión posdesastre. En este sentido, es recomendable que la comunidad participe en las siguientes actividades:

a) Decisiones de recuperación y reconstrucción para lograr que todos los intereses, necesidades y expectativas estén incluidas en los proyectos de desarrollo y reactivación y que se valoren más los objetivos y beneficios a largo plazo por sobre actividades cortoplacistas que beneficien a un grupo minoritario.

b) Búsqueda de diversas fuentes de financiamiento.

c) Mejorar el desarrollo económico y la resiliencia y eficacia de la comunidad a través del aprovechamiento de las oportunidades de reconstrucción y recuperación.

d) Considerar las oportunidades de protección, conservación y recuperación del medioambiente y recursos naturales para optimizar las funciones de la naturaleza al mismo tiempo que se extienden los beneficios hacia la comunidad.

Muchas tareas, como verificar la información, encuestas y entrevistas, requieren de la inclusión de la comunidad para determinar los aspectos materiales del

13. Charles Adie, Holistic Disaster Recovery: Ideas for Building Local Sustainability After a Natural Disaster. Pennsylvania: Diane Publishing. 2001 Recuperado de 〈http://www.preventionweb.net/publications/ view/1746>. 
caso. Según Mileti ${ }^{14}$ los stakeholders o actores sociales ${ }^{15}$ deben colaborar con la creación de política pública para reducir la posibilidad de conflictos durante la implementación y desarrollo de los planes.

5. Uno de los retos es lograr compartir información entre los actores públicos, privados y del sector involucrados para alcanzar mayor eficiencia. Para Marjanovic y Hallikainen ${ }^{16}$ uno de los retos en el proceso de recuperación es compartir el conocimiento desde los diferentes contextos del proceso, los límites de la organización y los profesionales. Por otra parte, la provisión de presupuestos, consultoría y el proceso de adquisiciones son más eficientes cuando existe la suficiente información y está disponible para las diferentes dependencias del gobierno, ONG y entidades privadas.

6. El gobierno y demás organizaciones tienen el mismo rol al momento de implementar un proyecto, actividad que requiere cierto nivel de autoridad y responsabilidad. Se sugiere contratar a personal capacitado a tiempo completo para prevenir la falta de recursos humanos calificados durante la implementación de los diferentes proyectos de reconstrucción y recuperación de la zona; se requiere que exista una clara asignación de responsabilidades y roles para realizar el proceso de contratación de personal especializado. ${ }^{17}$

Hay que considerar que cada persona puede verse afectada, positiva o negativamente, por una decisión gubernamental o de una agencia de ayuda humanitaria o desarrollo, por lo que es de vital importancia incluir a todos los actores en el proceso de toma de decisiones; como estrategia, se sugiere construir una base de actores amplia e inclusiva.

7. En la evaluación del daño hay dos momentos en que se debe recoger la información necesaria para realizar la evaluación de daños: una visita in situ después del suceso, donde se utiliza como herramienta la observación, y un segundo

14. Dennis Mileti, Disaster by Design: A Reassessment of Natural Hazards in the United States (Washington D. C.: Joseph Henry Press, 1999).

15. Los stakeholders o actores sociales "pueden ser personas, grupos u organizaciones que tienen interés en un proyecto o programa. Los actores claves son usualmente considerados como aquellos que pueden influenciar significativamente (positiva o negativamente una intervención) o son muy importantes para que una situación se manifieste de determinada forma". Esteban Tapella, El mapeo de actores claves (Córdoba: Universidad Nacional de Córdoba/Inter-American Institute for Global Change Research, 2007).

16. Olivera Marjanovic y Petri Hallikainen, "Disaster Recovery-New Challenges and Opportunities for Business Process Management Research and Practice", Pacific Asia Journal of the Association for Information Systems, vol. 5 (2013). Disponible en 〈http://aisel.aisnet.org/pajais/vol5/iss1/3/〉.

17. Natt Leelawat, Anawat Suppasri y Fumihiko Imamura, "Disaster Recovery and Reconstruction Following the 2011 Great East Japan Earthquake and Tsunami: A Business Process Management Perspective", International Journal of Disaster Risk Science, No. 3 (2015): 310-4. 
momento donde se hacen entrevistas y encuestas a las personas afectadas. Toda la información recolectada servirá para determinar si es necesario establecer una estrategia de recuperación o de reconstrucción; al mismo tiempo que servirá para evaluar rápidamente los objetivos de recuperación. ${ }^{18}$

8. Finalmente, para lograr la reactivación económica, Carter recomienda:

a) La gestión de desastres debe incorporar, entre sus procesos, la comprensión de dónde y cómo las agencias deben comprometerse y participar para apoyar el desarrollo de los mercados existentes y nuevos y analizar los costos y beneficios de un marco conceptual apropiado de intervención.

b) Reformar el análisis de los mercados antes y después de las crisis a través de la evaluación de los mercados y el estudio de los sistemas de mercado para ampliar la base de los agentes del mercado.

c) Construcción de relaciones entre las agencias de ayuda humanitaria y la asistencia técnica en temas de desarrollo -económico, microfinanciero, productivo- para visibilizar y fortalecer las sinergias en el ordenamiento de los mercados.

d) Mejorar la colaboración y el trabajo en conjunto entre las agencias de ayuda humanitaria y el sector privado. ${ }^{19}$

\section{IMPLEMENTACIÓN DE POLÍTICA PÚBLICA DESDE LOS ACTORES}

Para Joan Subirats, una política pública es "un conjunto de decisiones y acciones que resultan de interacciones repetidas entre actores públicos y privados, cuyas conductas se ven influenciadas por los recursos que disponen y por las reglas institucionales generales (concernientes al funcionamiento global del sistema político) y especificas (propias al ámbito de intervención en cuestión)". ${ }^{20}$

Toda intervención pública está encaminada a cambiar el estado "natural" de las cosas en la sociedad, por vía de unas determinadas acciones que generan impactos o efectos. Los efectos o impactos que se pretende generar con cada política pública suelen estar contenidos en los objetivos de la misma y constituyen la razón de su existencia.

18. Ibíd.

19. Becky Carter, Economic and Market Resilience before and After Shocks. Governance, Social Development, Humanitarian, Conflict (GSDRC) (Birmingham: GSDRC/University of Birmingham, 2016). Disponible en 〈http://www.gsdrc.org/wp-content/uploads/2016/02/HDQ1328.pdf〉.

20. Joan Subirats y otros, Análisis y gestión de politicas públicas, vol. 1 (Barcelona: Ariel, 2008), 23. 
Todo individuo o grupo social con un cierto grado de vinculación con el problema colectivo que origina la política pública debe considerarse actor potencial del "espacio" de dicha política. Ahora bien, la participación directa o indirecta de los actores en la política pública dependerá, entre otros elementos, del grado de conciencia que el actor tenga respecto de sus propios intereses, de su capacidad recursiva y de la construcción de coaliciones para defender sus derechos, así como de su decisión estratégica de pasar a la acción o mantenerse fuera del espacio de toma de decisiones. ${ }^{21}$

Se puede distinguir a los actores de las políticas públicas en función de su carácter público, es decir, los actores político-administrativos -sean nacionales o internacionales-, o de su carácter no público, es decir, los actores que pertenecen a las esferas que se puede denominar socio-económica y socio-cultural. Los actores privados pueden dividirse a su vez en grupos-objetivo (actores cuyo comportamiento se define políticamente como la causa (in)directa del problema), beneficiarios finales de una política pública (actores que sufren los efectos negativos del mencionado problema y cuya situación pretende mejorarse a través de la intervención pública) y grupos terceros, afectados de manera indirecta por la política pública, ya sea de manera positiva (beneficiarios), o de manera negativa (afectados), reuniendo por tanto ambas categorías a todos los actores cuya situación personal se ve modificada por la política pública aun cuando la misma no los hubiera identificado. ${ }^{22}$

A partir del marco teórico propuesto, este artículo busca resaltar la necesidad de intervención directa de los actores en todas las fases de política pública de reconstrucción productiva de las zonas afectadas por el terremoto de 16 de abril de 2016. Para ello, se partirá de lo previsto en la Constitución de la República 2008 y en la normativa secundaria respecto de la participación de los actores en la co-construcción de política pública, tanto en el caso de sus economías individuales como frente a situación de excepción como el caso de los efectos del terremoto en sus vidas y las de sus familias.

21. Peter Knoepfel, Larrue Corinne y otros, "Hacia un modelo de análisis de políticas públicas operativo: Un enfoque basado en los actores, sus recursos y las instituciones", Ciencia Política, Perspectivas Teóricas, No. 3 (2007).

22. Joan Subirats y otros, Análisis y gestión de políticas públicas. 


\section{POLÍTICA PÚBLICA PREVISTA EN LA CONSTITUCIÓN Y EN LA LEGISLACIÓN ECUATORIANA}

\section{SOBRE DESASTRES NATURALES}

El artículo 389 de la Constitución Política del Ecuador de 2008 prevé que "el Estado protegerá a las personas, las colectividades y la naturaleza frente a los efectos negativos de los desastres de origen natural o antrópico mediante la prevención ante el riesgo, la mitigación de desastres, la recuperación y mejoramiento de las condiciones sociales, económicas y ambientales, con el objetivo de minimizar la condición de vulnerabilidad".

El mismo artículo señala que el Estado es quien ejerce la rectoría del sistema nacional descentralizado de gestión de riesgo a través del organismo técnico establecido en la ley. Por su parte, la Ley de Seguridad Pública y del Estado establece que la Secretaría Nacional de Gestión de Riesgos es el órgano rector del sistema nacional descentralizado de gestión de riesgo.

Al día siguiente del terremoto, el Presidente del Ecuador emitió:

- El Decreto 1001, que declara el estado de excepción en las provincias de Esmeraldas, Manabí, Santa Elena, Santo Domingo de los Tsáchilas, Los Ríos y Guayas, por los efectos adversos del desastre natural, dispone la movilización nacional en esas provincias y ordena que se sitúe los fondos necesarios para atender la situación de excepción.

- El Decreto 1002, de 18 de abril de 2016, amplia el primero en el sentido de que la movilización es para todo el territorio nacional, y además dispone las requisiciones a las que haya lugar para solventar la emergencia producida. El Decreto 1003, de 23 de abril de 2016, declara Duelo Nacional durante ocho días por la pérdida de vidas humanas.

- El Decreto 1004, de 26 de abril de 2016, crea el Comité para la Reconstrucción y Reactivación Productiva y del Empleo en las zonas afectadas por el terremoto. Dicho Comité está integrado por el vicepresidente de la República, el secretario nacional de Planificación, el ministro coordinador de Desarrollo Social, el ministro coordinador de Producción, Empleo y Competitividad, el ministro coordinador de Seguridad Interna y Externa, el prefecto de Manabí, y dos alcaldes en representación de las zonas afectadas. Se admite la asistencia de un delegado de las Cámaras de la Producción de Manabí, y otro delegado de los pequeños comerciantes y empresarios de la provincia de Manabí. 
La primera crítica que cabe a la conformación de este comité es la ausencia de delegados de los afectados, que son actores principales por ser los afectados-beneficiarios de la política que se aplicará.

La coordinación de la gestión de emergencias, es decir, "la organización y la gestión de los recursos y las responsabilidades para abordar todos los aspectos de las emergencias, especialmente la preparación, la respuesta y los pasos iniciales de la rehabilitación", ${ }^{23}$ en Ecuador se coordina a través de los Comité de Operaciones de Emergencia (en adelante COE) a nivel nacional, provincial y cantonal. Las instituciones públicas que realizaron acciones en territorio son: Cuerpo de Bomberos de Quito, Fuerzas Armadas, Ministerio del Interior, Policía Nacional y Secretaría de Gestión de Riesgos.

Así mismo, por iniciativa del Ejecutivo, la Asamblea Nacional promulgó la Ley Orgánica de Solidaridad y Corresponsabilidad Ciudadana para la Reconstrucción y Reactivación de las Zonas Afectadas, ${ }^{24}$ norma que propuso medidas para obtener financiamiento para las actividades de recuperación integral, tales como:

- El aporte de un día de sueldo para quienes perciben más de mil (1.000) dólares, hasta el máximo de 5 días en caso de que el trabajador perciba cinco mil (5.000) dólares al mes;

- Incremento del IVA en dos puntos porcentuales: del 12 al 14\%;

- Contribución del 3\% adicional sobre las utilidades de las empresas, y contribución del $0,9 \%$ a las personas naturales con patrimonio mayor a un millón de dólares.

Se han dictado además normas tributarias, laborales, de crédito, de vivienda, entre otras, que atienden a la situación de los damnificados y prevén beneficios, exenciones o ampliación de plazos y flexibilización de condiciones para el cumplimiento de obligaciones de los habitantes de las provincias de Manabí y Esmeraldas, que han sido afectados por el terremoto.

El último informe, presentado el 30 de agosto de 2016 por el ministro de Finanzas del Ecuador y el secretario técnico del Comité de Reconstrucción y Reactivación Productiva ante la Asamblea Nacional, indica que hasta el momento se han asignado 888 millones, de los cuales 182 millones han sido devengados. Los recursos entregados para vivienda, reparación y construcción, así como la atención a las familias alberga-

23. UNISDIR 2009: Terminología sobre la reducción de riesgos de desastres (Naciones Unidas: UNISDIR, 2009), 18. Disponible en 〈https://www.unisdr.org/files/7817_UNISDRTerminologySpanish.pdf〉.

24. R. O.-S. 759, de 20 de mayo de 2016. 
das en Manabí y Esmeraldas son los más elevados. También se ha destinado un fondo importante para vialidad y educación.

Se han definido 18 reasentamientos poblacionales para instalar a las familias que vivían en zonas declaradas de riesgo, lo que ha generado denuncias de que algunos de esos reasentamientos responden a otros intereses; esto ha pasado en ciudades como Muisne o Canoa. De responder a la realidad las denuncias, es decir, que en algunos casos los reasentamientos se debieron a otros intereses y no a salvaguardar la vida de quienes vivían antes en esos territorios, sería muy grave, pues significaría el aprovechamiento de una situación límite para obtener beneficios. En todo caso, lo que es indudable es la falta de comunicación y acuerdo entre el gobierno y los afectados, quienes, además de sufrir los estragos del terremoto, ahora son obligados, en algunos casos por la fuerza, a dejar sus tierras y a vivir en otros lugares.

También hay denuncias de utilización de los fondos para la reconstrucción con otros fines. Por estos motivos, se ha solicitado al Gobierno que se cree una Comisión de Vigilancia del manejo del presupuesto para reconstrucción, algo que no ha tenido eco en el Ejecutivo.

\section{SOBRE LA ECONOMÍA SOLIDARIA}

A más de un año del terremoto es preciso evaluar la política pública de recuperación económica y productiva en las provincias más afectadas. Para ello, es importante tener a la vista la estructura económica prevista en la Constitución.

La Constitución Política ecuatoriana prevé un cambio de paradigma respecto del modelo económico. Así, el artículo 283 determina que la economía ecuatoriana es social y solidaria, y señala algunos principios que ayudan a definirla:

El sistema económico es social y solidario; reconoce al ser humano como sujeto y fin; propende a una relación dinámica y equilibrada entre sociedad, Estado y mercado, en armonía con la naturaleza; y tiene por objetivo garantizar la producción y reproducción de las condiciones materiales e inmateriales que posibiliten el buen vivir. ${ }^{25}$

Grijalva y Trujillo sostienen que, en la economía social y solidaria que proclama la Constitución de 2008, el mercado pierde centralidad en el sistema económico, para convertirse en un mecanismo más, junto con otras formas de intercambio justo y de organización económica, no siendo el ánimo de lucro el móvil del sistema económico

25. Inciso primero del art. 283 de la Constitución de la República del Ecuador. 
sino la armonía en las relaciones de los seres humanos entre sí, con la naturaleza y las futuras generaciones. ${ }^{26}$

De acuerdo con estos autores, el sistema económico que se plantea en la vigente Constitución no es el de una economía planificada o estatista, ya que reconoce otras formas de organización económica, aunque regula fuertemente al mercado. ${ }^{27}$ En este punto, Angélica Porras ${ }^{28}$ sostiene que se deben considerar las implicaciones que tiene la noción prevista en nuestra Constitución sobre el sumak kawsay, que tiene importantes vinculaciones con el concepto de trabajo. Su tesis indica:

1. El sumak kawsay informa y justifica toda la Constitución, y por tanto no puede ser reducida a los Derechos Económicos, Sociales y Culturales, o al Régimen del Buen Vivir contenidos en la Constitución, como se ha venido haciendo.

2. El sumak kawsay, en cuanto a lo social y político, busca la superación de la colonialidad y la construcción de una sociedad más relacional, responsable y respetuosa de las diferencias.

3. El sumak kawsay, en cuanto a lo económico, supone tres transformaciones trascendentales:

a) Un cuestionamiento al modelo de desarrollo y su matriz productiva.

b) Una nueva relación trabajo-capital.

c) Una nueva relación naturaleza-ser humano. ${ }^{29}$

Es preciso aclarar, aunque sea de forma sucinta, en qué consiste el sumak kawsay:

El Buen Vivir andino (sumak kawsay) se establece como un consolidado comunitario en cuyo tejido, y para mantenerlo con-viven seres diferentes en una calidad de mingados, es decir, de seres (no solo humanos) que laboran desde y para el mantenimiento y reproducción de esa condición y calidad de vida. Humanos, plantas, animales y tierras son comunidad que se revitaliza incesantemente, complementándose, correspondiéndose, reciprocándose, es decir, relacionándose, dando lugar no solo al acuerdo, son al desacuerdo y a lo contrario, sin lo cual no podría tener un sentimiento de ciclicidad dinámica, pues lo andino

26. Agustín Grijalva y Julio César Trujillo, "El fundamento constitucional de la nueva economía", La Tendencia 10 (marzo 2010). Consulta: junio de 2017. Disponible en 〈http://repositorio.flacsoandes.edu.ec/ handle/10469/4621\#.WohNbq7iZ0w».

27. Ibíd.

28. Angélica Porras Velasco, "Constitución, sumak kawsay y trabajo", en Ramiro Ávila, comp., Estado, Derecho y Justicia. Estudios en honor a Julio César Trujillo (Quito: Universidad Andina Simón Bolívar, Sede Ecuador/Corporación Editora Nacional, 2013).

29. Ibíd. 
es lo uno y lo otro, lo de arriba y lo de abajo, lo de un lado y lo de otro, representando al conjunto espacial asumido como Madre. ${ }^{30}$

La Carta Fundamental recupera este concepto desde un ejercicio de diálogo plural, en el que se recogen importantes demandas del movimiento indígena, los pueblos afroecuatorianos, así como otros movimientos sociales, tales como los feministas, ambientalistas, juveniles, entre otros. Entre las principales propuestas innovadoras del concepto, está la ruptura con el paradigma desarrollista y con los procesos de acumulación material y explotación de la naturaleza, así como con la reducción del Estado impulsada por el neoliberalismo, y con la desigualdad social productiva y distributiva. $^{31}$

Para alcanzar el objetivo final del sumak kawsay, la Constitución impulsa una relación diferente entre Estado, mercado, sociedad y naturaleza, reconociendo el sistema económico como social y solidario, un sistema que subordina al mercado a la vida humana y natural.

En ese sentido, también han ido evolucionando los Planes Nacionales del Buen Vivir (PNBV), que en su primera versión de 2007-2009, hablaba de la iniciativa privada como motor de la economía. El Plan Nacional de Desarrollo para el período 2009$2013^{32}$ evoluciona respecto del primero, en el sentido de tener como una de sus principales estrategias la democratización de los medios de producción, (re)distribución de la riqueza y diversificación de las formas de propiedad y organización. Estableciendo además que, "el escenario socioeconómico debe proyectarse como la combinación adecuada de formas organizativas que impulsen la economía social y solidaria y el reconocimiento de la diversidad en el sistema económico (formas de producción y comercialización, formas de propiedad) para asegurar su presencia activa en la representación y participación social en el escenario público de los próximos años", ${ }^{33}$ con especial énfasis en el sector agropecuario.

En el PNBV 2009-2013 se reconoce que el Estado debe auspiciar las formas alternativas de organización social y económica, la consolidación de asociaciones y cooperativas, apoyar el trabajo para el autoconsumo, el intercambio democrático y

30. Cecilia M. Andrade y otros, Cosmovisión andina, Sumak Ally Kawsay y economía comunitaria. Diálogos sobre Economía Social y Solidaria en Ecuador (Bilbao: Universidad del País Vasco, 2014).

31. Ibíd.

32. Que de acuerdo al art. 280 de la CE "es el instrumento al que deben sujetarse las políticas, programas y proyectos públicos; la programación y ejecución del presupuesto del Estado; y la inversión y asignación de los recursos públicos; y coordinar las competencias exclusivas entre el Estado central y los gobiernos autónomos descentralizados".

33. Plan Nacional del Buen Vivir 2009-2013, 102. 
el acceso adecuado de la ciudadanía en general a los bienes y servicios producidos. ${ }^{34}$ Contempla como décimo primer objetivo, la necesidad de establecer un sistema económico, social, solidario y sostenible, debiendo las acciones del Estado dirigirse a consolidar ese sistema, en el cual la planificación nacional y las intervenciones estatales permitan la transformación progresiva de la matriz productiva, a través de las etapas planteadas en la Estrategia para el Buen Vivir. ${ }^{35}$

Con el afán de tener continuidad, el Plan Nacional de Desarrollo 2013-2017 establece, como octavo objetivo, la consolidación del sistema económico social y solidario, de forma sostenible ${ }^{36}$ para ello plantea una serie de políticas y lineamientos estratégicos. ${ }^{37}$

Por su parte, la Ley Orgánica de la Economía Popular y Solidaria y del Sistema Financiero Popular y Solidario ${ }^{38}$ contiene objetivos y principios que, junto con lo anterior, constituyen el marco ideológico que el Gobierno ha previsto como sustento de su política pública en materia de Economía Solidaria. La Ley, en su artículo 4 enuncia una serie de principios que deben guiar la actuación de las personas y organizaciones de la economía popular y solidaria, siendo estos:
a) La búsqueda del buen vivir y del bien común;
b) La prelación del trabajo sobre el capital y de los intereses colectivos sobre los individuales;
c) El comercio justo y consumo ético y responsable:

34. Ibíd., 102 y 103.

35. Ibíd., 330 .

36. Ibíd., 247.

37. Estas políticas y lineamientos estratégicos son los siguientes:

a) Invertir los recursos públicos para generar crecimiento económico sostenido y transformaciones estructurales.

b) Consolidar el papel del Estado como dinamizador de la producción y regulador del mercado.

c) Fortalecer el manejo sostenible de las finanzas públicas.

d) Fortalecer la progresividad y la eficiencia del sistema tributario.

e) Afianzar la sostenibilidad de la balanza de pagos.

f) Mantener la sostenibilidad biofísica de los flujos económicos.

g) Garantizar una adecuada gestión de la liquidez para el desarrollo y para administrar el esquema monetario vigente.

m) Minimizar el riesgo sistémico de la economía.

i) Profundizar las relaciones del Estado con el sector popular y solidario.

j) Articular la relación entre el Estado y el sector privado.

Plan Nacional del Buen Vivir 2013-2017, 264 y s.

38. Publicada en el R. O. No. 444, de 10 de mayo de 2011. 
d) La equidad de género;

e) El respeto a la identidad cultural;

f) La autogestión;

g) La responsabilidad social y ambiental, la solidaridad y rendición de cuentas; y

h) La distribución equitativa y solidaria de excedentes.

A partir del marco normativo previsto, parece que la economía solidaria responde de mejor manera a los desafíos que la recuperación económica de las zonas afectadas por el terremoto presentan, y es que por vía de procesos asociativos y solidarios es mucho más sencillo no solo superar las pérdidas económicas que el terremoto provocó, sino incluso mejorar los niveles de vida y productividad anteriores al terremoto.

\section{LINEAMIENTOS PARA UNA PROPUESTA DE POLÍTICA PÚBLICA DE REACTIVACIÓN ECONÓMICA DE MANABÍ}

\section{Situación de Manabí antes y después del terRemoto}

En contexto, la provincia de Manabí, de acuerdo con el último censo del año 2010, tiene 1'345.779 habitantes, lo que representa el 9,4\% de los habitantes del Ecuador. El $63 \%$ de las personas vive en zonas urbanas, y el 37\% en zonas rurales. La Población Económicamente Activa (PEA) de Manabí asciende al 28\%, y según los datos del censo 2010 representa el $8 \%$ de la fuerza laboral del país. Su aporte económico a nivel nacional representa cerca del $5 \%{ }^{39}$

La distribución de la PEA por actividad económica en la provincia es la siguiente: el 37,4\% de la PEA de la provincia de Manabí se dedica a la agricultura, silvicultura, caza y pesca, siendo esta la actividad de mayor importancia para la provincia. La actividad que le sigue es el comercio que representa el 18,8\% de la PEA. También tienen gran importancia las actividades de servicios $(14,4 \%)$ y manufactura $(9,2 \%)$. Las actividades no bien especificadas alcanzan los $9 \%$, seguidas por la rama de la construcción que representa el 5,8\% y transporte 5,5\% de la PEA. Mientras que los establecimientos financieros representan apenas el $0,2 \% .{ }^{40}$

Estas actividades se han desarrollado mediante iniciativas personales, familiares o de empresas, lo que ha generado, en el caso de pequeños emprendimientos, poca productividad; y, en los emprendimientos grandes, una acumulación que no ha facilitado

39. Resultados del Censo 2010 de población y vivienda en Ecuador.

40. Ibíd. 
el mejoramiento de las condiciones socioeconómicas de la mayoría de la población, sino de grupos pequeños. A eso hay que sumar la acumulación de la propiedad de la tierra, que en buena parte del territorio de la provincia son latifundios que están en pocas manos.

Al revisar los datos de la economía solidaria, tenemos que en el país, hasta el 29 de junio de 2015, existían 6.816 organizaciones de la economía solidaria, de las cuales, 5.852 pertenecen al sector real o no financiero, el resto son organizaciones del sector financiero. Del número de organizaciones del sector real, ${ }^{41} 535$ se encontraban en la provincia de Manabí, representando un poco menos del 10\% del total nacional. Esas 535 organizaciones de la economía solidaria del sector real en Manabí eran principalmente asociaciones de producción (272), y las restantes se distribuían entre asociaciones de consumo, servicios y cooperativas de producción, servicios y vivienda. ${ }^{42}$

Desde el día después del terremoto hasta la primera semana de febrero de 2017, se crearon en Manabí 281 organizaciones de la economía social del sector real, que es una cifra bastante elevada si se miran las registradas antes de esa fecha. De las 281, 275 son asociaciones, y, de ese número, 205 son asociaciones de producción. ${ }^{43}$

De la información proporcionada se puede ver un rápido crecimiento de las organizaciones de la economía solidaria en Manabí después del terremoto, sobre todo de las asociaciones, para las que si bien se exigen los mismos requisitos ${ }^{44}$ de número de socios (10), y un monto de aporte inicial al fondo social (un salario básico unificado que el año 2017 estaba en 375 dólares) que para las cooperativas, no se requiere contar con otros requisitos, tales como informes favorables de autoridad competente; además, el control es mayor en el caso de las cooperativas.

Este escenario debe ser considerado como apropiado para implementar una política pública que fomente y fortalezca al sector de la economía solidaria, para que las organizaciones que se han creado tengan una verdadera posibilidad de subsistencia, de éxito, y puedan convertirse en referentes para el resto del país.

41. Entre las que se cuentan cooperativas, asociaciones y organizaciones comunitarias.

42. 〈https://servicios.seps.gob.ec/reps-internet-web/paginas/consultarOrganizaciones.jsf〉.

43. 113 de esas asociaciones de producción son textiles. En menor medida se han creado asociaciones de producción agrícola y de pesca.

44. Art. 7 Reglamento a la Ley de Economía Popular y Solidaria. Decreto Ejecutivo 1061, publicado en el R. O.-S. 648, de 27 de febrero de 2012. 


\section{LINEAMIENTOS PARA UNA POLÍTICA PÚBLICA PARA EL DESARROLlO productivo de Manabí a través de la Economía Solidaria}

Para lograr un real involucramiento en el proceso de recuperación, una política pública sobre desarrollo productivo posdesastre debe integrar satisfactoriamente a todos los organismos, instituciones, gremios y comunidades afectadas en las fases de planificación, gestión y evaluación de la política pública.

Para que la construcción de las políticas públicas necesarias para alcanzar los objetivos propuestos sea realizada corresponsablemente, se busca que todos los procesos de rehabilitación y recuperación de la productividad cuenten con la participación activa de los grupos de interés.

El Comité de Reconstrucción y Reactivación Productiva debería ser el organismo que coordine el proceso de rehabilitación y recuperación productiva, para evitar la superposición y duplicación de esfuerzos, así como para asegurar el uso ineficiente de recursos.

La fase de planificación de la política pública debió haber iniciado por la identificación de las actividades productivas históricas: turismo, pesca, comercio, acuicultura, agricultura, y de las nuevas que puedan implementarse, para la ejecución de talleres de capacitación por parte de las universidades y sector productivo de la región. Esta capacitación permite que los recursos a ser invertidos sean utilizados de manera eficiente y rápida.

Este mapeo de las actividades productivas y el acceso a talleres y capacitaciones también deberían ser consideradas una tarea del gobierno, y, por lo tanto, la respectiva política pública debería implementarse conjuntamente con la ayuda técnica del sector privado, organismos de la sociedad civil y representantes de los propios interesados. Así, es necesario crear programas a través de alianzas público-privadas para desarrollar y fortalecer capacidades en temas de emprendimientos solidarios, economía popular y solidaria, comercio justo, turismo sustentable, mecanismos de acceso al crédito, tejido social y organización, diseño de proyectos, capital social y resiliencia.

La realidad nos muestra que se ha creado un número significativo de asociaciones productivas en Manabí después del terremoto; algunas en actividades tradicionales de la zona, como agricultura, acuacultura y pesca, y un buen número para la realización de actividades textiles, que son nuevas, por lo menos para ser realizadas en asociación.

Debido a que este tipo de organizaciones tienden a ser más débiles que las cooperativas, es necesario que se fomente la organización de segundo y tercer piso de esas asociaciones, es decir, federaciones y confederaciones, para tener mejores expectativas de sobrevivencia y de éxito en las actividades productivas que van a realizar. Otro elemento importante es la promoción de los principios cooperativos como requisito 
pre y posorganizativo del sector solidario, y concretamente del que existe en Manabí. Es preciso trabajar en el empoderamiento de los actores de la economía solidaria, para que dejen de mirarse como víctimas o meros beneficiarios de ayudas por parte del Estado, y se conviertan en verdaderos gestores del cambio, primero de sus propias realidades, y también de superación de un sistema excluyente a uno solidario e inclusivo.

Haciendo un análisis de la respuesta al desastre (acápite "Respuesta al desastre" del presente artículo), es evidente que para lograr una reactivación productiva, teniendo como eje de acción la economía solidaria, se deben identificar las demandas sociales y políticas de la población y enfocarse en la provisión de recursos financieros, en el desarrollo de capacidades, en el fortalecimiento del tejido social y asociativo, y en el involucramiento de la comunidad con una adecuada gobernanza e institucionalidad.

Se deben desarrollar y promover estrategias de financiamiento que tengan como beneficiarias tanto a las organizaciones sociales de base como a las de segundo y tercer piso, las cuales podrían beneficiarse de los créditos, con ciertas condiciones de asociatividad entre organizaciones del mismo tipo.

Es necesario contar con una política pública de edificaciones, tanto para vivienda como para el desarrollo de actividades productivas y de mercado, que cumpla con los criterios de sustentabilidad tanto para materiales como para seguridad, promoviendo el uso de materiales de la zona para la construcción de las nuevas edificaciones y viviendas, considerando la compatibilidad de uso de suelo e impacto ambiental. Esta política pública puede responder también a la creación de empleo y al desarrollo de cultivos apropiados para ser utilizados como material de vivienda.

La provincia de Manabí cuenta con áreas naturales dentro del Sistema Nacional de Áreas Protegidas, una política pública adecuada podría promover el turismo sustentable y comunitario, lo cual también ayudaría a reactivar la economía de la zona, al mismo tiempo que se protege y conserva el medioambiente y las tradiciones culturales ancestrales de la zona de la antigua cultura Jama-Coaque. Las políticas de promoción turística deben construirse conjuntamente con la planificación territorial y de reconstrucción de hoteles y gastronomía.

Es preciso legalizar las tierras en la provincia de Manabí, lo que permitirá ejecutar las políticas públicas encaminadas al desarrollo productivo de la zona. El proceso de legalización pasa en algunos casos por el reconocimiento jurídico de la propiedad, en otros por la ejecución de reasentamientos, y, en general, por proveer de espacios físicos adecuados donde las personas puedan desarrollar los proyectos de vida y productivos en condiciones de dignidad. Para ello, es preciso trabajar en una planificación territorial en la que se prevea también el crecimiento demográfico, se cuiden las fuentes de agua, las redes de alcantarillado, el alumbrado público, transporte, gestión de residuos y zonas de uso público como mercados, parques, malecón, entre otros. 
Finalmente, es importante conformar una comisión de veeduría y control del manejo y gestión de los recursos para cumplir los principios de transparencia y rendición de cuentas.

\section{CONCLUSIONES}

El país, a pesar de contar con una Secretaría Técnica de Gestión de Riesgo, no está listo para afrontar desastres de gran envergadura; como se desprende del análisis del marco legal, fue necesario que el Ejecutivo emita Decretos para atender de manera urgente las principales necesidades y requerimientos de los afectados.

Es indispensable construir política pública para la gestión de desastres antes y después, e involucrar a la comunidad afectada en su construcción.

Las alianzas con el sector privado, sea este lucrativo o no lucrativo, son necesarias para lograr mejores resultados, y, como se ha dicho, el momento actual es muy apropiado para trabajar en esas alianzas que vinculen a la economía solidaria.

Para lograr una reactivación económica adecuada se necesita tanto la voluntad política como un adecuado levantamiento de información para conocer las actividades productivas tradicionales de la zona, desarrollar el fortalecimiento de capacidades, y crear el ambiente propicio para que el mercado funcione.

El diagnostico indica que la propuesta de política pública debe promover la eficiencia del mercado y de los negocios, el desarrollo económico local y la integración económica de la zona por medio de la asociación con fines productivos, de personas y organizaciones con similares necesidades e intereses, que por medio de proyectos productivos solidarios mejoren sus economías y aporten al mejoramiento de la zona donde se encuentran, a través de la oferta de bienes y servicios de calidad, elaborados y ofrecidos con estándares de solidaridad y sustentabilidad.

Después de un desastre natural, la comunidad, el medioambiente y el mercado, tienen que reponerse del shock, lo que debe entenderse como un proceso de creación y desarrollo de resiliencia para poder afrontar el proceso de recuperación integral y estar preparados para un nuevo desastre. Es evidente la necesidad de fortalecer el tejido social de la zona mediante la creación de asociaciones de la economía popular y solidaria para reactivar el sector productivo, fomentando la organización de segundo y tercer nivel de dichas asociaciones. Se debe trabajar desde la base, con las comunidades para crear resiliencia ante los desastres naturales, teniendo en cuenta que las asociaciones de productores son más frágiles que las cooperativas.

Esta investigación abrió la posibilidad de realizar futuras investigaciones en territorio para trabajar en procesos asociativos y de cooperación en temas sociales de relevancia, como la provisión de servicio público de energía, agua, educación, desarrollo 
económico y fortalecimiento del tejido social por medio de la construcción de la resiliencia en la comunidad. Una investigación futura puede ser de corte etnográfico con el objetivo de identificar los obstáculos para formar asociaciones, determinar las razones por las cuales algunos grupos son excluidos de los procesos asociativos, fortalecer a las asociaciones ya constituidas; mejorar su acceso a servicios financieros, y mejorar los procesos productivos mediante la especialización de trabajo de los asociados.

La recomendación es que, en algunos casos, la reactivación productiva deberá iniciar con invitaciones a la asociatividad, y, en otros casos, cuando ya exista un proceso asociativo previo, trabajar en los valores y principios solidarios y cooperativos, hasta lograr asociaciones de productores o trabajadores que comparten similares necesidades e intereses y vean a su organización no solo como un medio para obtener unas mínimas ganancias que les permitan subsistir, sino como verdaderos motores de productividad y cambio de una realidad excluyente a una incluyente.

\section{BIBLIOGRAFÍA}

Adie, Charles. Holistic Disaster Recovery: Ideas for Building Local Sustainability After a Natural Disaster. Pennsylvania: Diane Publishing, 2001. Disponible en «http://www.preventionweb.net/publications/view/1746>.

Andrade, Cecilia M., y otros. "Cosmovisión andina, Sumak Ally Kawsay y economía comunitaria”. En Diálogos sobre Economía Social y Solidaria en Ecuador. Bilbao: Universidad del País Vasco, 2014.

Bagai, Dipa Singh. Tsunami Thailand: One Year Later: National Response and Contribution of International Partners. Bangkok: United Nations Country Team (UNCT), 2005. Disponible en 〈http://siteresources.worldbank.org/INTEASTASIAPACIFIC/Resources/TsunamiTH_One-Year-Later.pdf).

Carter, Becky. "Economic and Market Resilience before and After Shocks". En Governance, Social Development, Humanitarian, Conflict (GSDRC). Birmingham: GSDRC/University of Birmingham, 2016. Disponible en 〈http://www.gsdrc.org/wp-content/uploads/2016/02/ HDQ1328.pdf».

Carrio Carro, Luis. "El concepto de resiliencia y su aplicación en Avilés". Tesis de maestría, Universidad de Oviedo, Máster Universitario en Análisis y Gestión de Emergencia y Desastre, 2015. Disponible en 〈http://digibuo.uniovi.es/dspace/handle/10651/32011〉.

Cavallo, Eduardo, Andrew Powell y Oscar Becerra. "Estimating the Direct Economic Damages of the Earthquake in Haiti". The Economic Journal, No. 546 (2010).

Comisión Económica para América Latina y Caribe (CEPAL). The Natural Disaster of March 1987 in Ecuador and its Impact on Social and Economic Development. Santiago de Chile: CEPAL, 1987. Disponible en 〈http://repositorio.cepal.org/bitstream/handle/11362/35807/ S8740406_en.pdf?sequence=2\&isAllowed=y. 
Grijalva, Agustín, y Julio César Trujillo. "El fundamento constitucional de la nueva economía”. La Tendencia 10 (marzo 2010). Disponible en «http://repositorio.flacsoandes.edu.ec/ handle/10469/4621\#. WohNbq7iZ0w .

Jackson, Ruth, Daniel Fitzpatrick y Prabin Man Singh. Building Back Right: Ensuring Equality in Land Rights and Reconstruction in Nepal. Oxford: Oxfam, 2016. Disponible en 〈http://policy-practice.oxfam.org.uk/publications/building-back-right-ensuring-equalityin-land-rights-and-reconstruction-in-nepal-606028.

Jara Vásquez, María Elena. "Constitución económica ecuatoriana y desarrollo”. En Estado, Derecho y Justicia. Estudios en honor a Julio César Trujillo. Quito: Universidad Andina Simón Bolívar, Sede Ecuador/Corporación Editora Nacional, 2013.

Knoepfel, Peter, Larrue Corinne y otros. "Hacia un modelo de análisis de políticas públicas operativo: Un enfoque basado en los actores, sus recursos y las instituciones". Ciencia Política, Perspectivas Teóricas, No. 3 (2007).

Lavell, Allan. "Sobre la gestión del riesgo: Apuntes hacia una definición". Scripta Nova-Revista (2001). Disponible en 〈http://microseguros.net/seminario/biblioteca/vulnerabilidad/ doc15036-lavell-gestion-riesgo.pdf〉.

Marjanovic, Olivera, y Petri Hallikainen. "Disaster Recovery-New Challenges and Opportunities for Business Process Management Research and Practice". Pacific Asia Journal of the Association for Information Systems, vol. 5 (2013). Disponible en 〈http://aisel.aisnet. org/pajais/vol5/iss1/3/>.

Márquez Domínguez, Juan, y Adriano Rovira Pinto. "El desarrollo local: una propuesta social y económica con fuerte base territorial”. Espacio y Desarrollo, No. 14 (2002).

Mileti, Dennis. Disaster by Design: A Reassessment of Natural Hazards in the United States. Washington D. C.: Joseph Henry Press, 1999.

Natt Leelawat, Anawat Suppasri y Fumihiko Imamura. "Disaster Recovery and Reconstruction Following the 2011 Great East Japan Earthquake and Tsunami: A Business Process Management Perspective”. International Journal of Disaster Risk Science, No. 3 (2015).

Porras Velasco, Angélica. “Constitución, sumak kawsay y trabajo”. En Estado, Derecho y Justicia. Estudios en honor a Julio César Trujillo. Quito: Universidad Andina Simón Bolívar, Sede Ecuador/Corporación Editora Nacional, 2013.

Secretaría de Gestión de Riesgos. Informe de Situación No. 17, de 19 de mayo de 2016. Disponible en 〈http://www.gestionderiesgos.gob.ec/wpcontent/uploads/downloads/2016/05/ INFORME-n71-SISMO-78-20302.pdf .

Secretaría Nacional de Planificación y Desarrollo, Evaluación de los costos de reconstrucción sismo en Ecuador. Abril 2016. Disponible en 〈http://www.planificacion.gob.ec/wpcontent/uploads/downloads/>.

Subirats, Joan, y otros. Análisis y gestión de políticas públicas, vol. 1. Barcelona: Ariel, 2008.

Tapella, Esteban. El mapeo de actores claves. Córdoba: Universidad Nacional de Córdoba/ Inter-American Institute for Global Change Research (IAI), 2007. 
UNISDR. Annual Report 2015 2014-15 Biennium Work Programme Final Report. Ginebra: UNISDR, 2015. Disponible en 〈http://www.unisdr.org/files/48588_unisdrannualreport2015evs.pdf〉.

Vargas, Jorge Enrique. Políticas públicas para la reducción de la vulnerabilidad frente a los desastres naturales y socio-naturales. Nueva York: Naciones Unidas, 2002.

Villanueva Haro, Benito. "Terremoto en Ica-Perú: por una reconstrucción con rostro social". Derecho y Cambio Social, No. 11 (2007). Disponible en 〈http://www.derechoycambiosocial.com/revista011/terremoto\%20en\%20ica.htm〉.

\section{Otros}

"Dos fuertes sismos sacuden Ecuador con epicentro en zona terremoto de abril". El Nuevo Herald. (Miami). 11 de julio de 2016. Disponible en 〈http://www.elnuevoherald.com/noticias/ mundo/americalatina/article88838672.html\#storylink=cpy〉.

Volver a la normalidad después del gran terremoto que azotó el este de Japón. Disponible en 〈http:/www.ilo.org/global/about-thilo/newsroom/features/WCMS_208802/lang--es/ index.htm〉. 